\title{
Multicenter Analysis of the Oncological Positivity on Restaging Resection (Re-Turbt) of Urothelial Non-Muscle-Invasive Bladder Cancer
}

\author{
Ubirajara Ferreira ${ }^{1}$, Wagner E. Matheus ${ }^{1, *}$, Wilmar Azal Neto ${ }^{1}$, Marcelo A. Souza ${ }^{1}$, Roni C. \\ Fernandes $^{2}$, Bruno N. Calado ${ }^{2}$, Gustavo B. Mendonça ${ }^{1}$, Leonardo Reis ${ }^{1}$, João L. G. Parizi ${ }^{3}$, \\ Stênio C. Zequi ${ }^{3}$ \\ ${ }^{I}$ State University of Campinas - UNICAMP - Campinas, SP - Brazil \\ ${ }^{2}$ Santa Casa de São Paulo School of Medical Sciences - São Paulo, SP - Brazil \\ ${ }^{3}$ A.C. Camargo Cancer Center - São Paulo, SP-Brazil
}

*Corresponding Author: Wagner Eduardo Matheus. State University of Campinas-UNICAMPCampinas, SP-Brazil, ORCID-0000-0003-0811-2616.Brazil,Email -wematheus@uol.com.br

\begin{abstract}
Objectives: The main objective was to determine the oncological positivity on restaging TURBT (re-TURBT), in patients submitted to a first complete transurethral resection of bladder tumor (TURBT). Moreover, to verify the most important site of tumor recurrence and changes in the treatment initially proposed.
\end{abstract}

Methods: We retrospectively reviewed the records of patients who underwent a re-TURBT for urothelial nonmuscle-invasive bladder cancer (NMIBC) between June 2011 and June 2014. Patients with known incomplete resections or muscle invasive tumor were excluded from the study.

Results: Of the 66 (sixty-six) patients who met the inclusion criteria, residual tumor was detected in 14 $(21.2 \%)$ at the re-TURBT. All of these patients had a Tl or high-grade tumor at initial TURBT. Only one $(1.5 \%)$ patient had a pathological finding on the re-TURBT (pT2) that changed the therapeutic strategy to radical cystectomy.

Conclusions: The positivity of re-TURBT was not so high like in previous studies, was detected only at the primary tumor site and did not change the therapeutic strategy. Complete resection and better choice of these patients are important to diminish the indications of the re-TURBT.

Abbreviations : TURBT: transurethral resection of bladder tumor, Re-TURBT: restaging transurethral resection of bladder tumor, BCa: bladder cancer, NMIBC: urothelial non-muscle-invasive bladder cancer, MM: muscularis mucosae, MP: muscularis propria, EAU: European Association of Urology, WLC: whitelight cystoscopy

Keywords: Bladder cancer; Transurethral resection; Urothelial carcinoma; Restaging TURBT; Re-TURBT

\section{INDRODUCTION}

Bladder Cancer $(\mathrm{BCa})$ is the second most common malignancy of the genitourinary tract and represents a significant cause of cancer morbidity and mortality [1]. Its incidence and prevalence have been increasing through the last years, although the mortality has decreased. The long course of disease, necessity of constant monitoring and morbidity from the required treatment surgeries are some of the factors responsible for the enormous costs involved with the care of patients with $\mathrm{BCa}$ [2].
In this scenario, authors have been increasingly studying various molecular markers that might be useful in an early diagnosis of initial $\mathrm{BCa}$ or its recurrence, some of these include p53, p21, $\mathrm{pRB}$ and p27. However, despite large efforts, they have yet to reach everyday practice $[2,3]$. It is a heterogeneous tumor ranging from the benign behaviour of a low-grade $\mathrm{Ta}$ to the aggressiveness of a high-grade invasive cancer; and so the understanding of these many variants is essential for us to better comprehend disease prognosis and therefore be able to choose the most appropriate treatment [4]. 
At the moment of presentation, $80-85 \%$ of $\mathrm{BCa}$ are restricted to the bladder, from those $85 \%$ are urothelial non-muscle-invasive bladder cancer (NMIBC). Formerly known as "superficial" bladder cancer which comprises Ta, T1 and Carcinoma in Situ (CIS) [5, 6]. Initial management is complete transurethral resection (TURBT) which provides accurate primary staging (tumor type, grade and depth of invasion) and supporting therapeutic decision. Endoscopic resection is a common urologic procedure and its goal is to perform it in a complete way. The surgeon has to report that all macroscopic tumors have been removed and the uropathologist has to confirm that lamina propria and muscularis propria were obtained [5, 7].

The histopathological analysis of $\mathrm{BCa}$ specimens after a TURBT differs notoriously from the usual histological evaluation of tumor samples, mainly due to the multiple fragments resected using continuous eletrocautery. Thus, the pathologist receives a specimen without anatomic orientation, has to process many fractions and deal with potentially extensive cautery artefact [1]. The most important information in this analysis is about invasion of the lamina propria or detrusor muscle (muscularis propria).

Despite the importance, it may be difficult for the pathologist to distinguish between muscularis mucosae (MM) and muscularis propria (MP) muscle bundles in some TURBT samples. When it is not possible to examine the detrusor layer or there is doubt in distinguishing MM from MP, the urologist must proceed with another TURBT for accurate verification of the pT stage [8]. Dalbagni et al revealed the absence of MP in $40 \%$ of T1 tumors, while Maruniak showed that $51 \%$ of the histological specimens had no MP $[9,10]$. The correct classification of this sub sequential procedure must be reviewed. The TURBT after incomplete resection due to factors such as multiplicity, size or location would be better called repeat resection. Restaging TURBT should be considered only if the primary resection was complete and ReTURBT is made to provide additional pathologic and disease information. According to the European Association of Urology (EAU) Guidelines 2014, the indications of re-TURBT are: if there is no MP in the specimen after initial resection, in all $\mathrm{T} 1$ tumors and in all high grade tumors. If there is a precise indication, it should be accomplished in 2-6 weeks, not only to accurately restage, but also to evaluate the presence of residual cancer, before a correct subsequent management decision is made.

This present study brings a series of patients with NMIBC submitted to a complete TURBT and re-TURBT of its primary site, and the main goal is to provide an analysis of data regarding the oncologic positivity on the pathological results of the second surgery. Moreover, to verify the most important site of tumor recurrence and changes in the treatment initially proposed.

\section{MATERIAL AND MeTHOdS}

A retrospective review was performed, including data from all 66 patients who underwent a complete initial TURBT and reTURBT for NMIBC, between June 2011 and June 2014, in three distinct tertiary medical centers, which are oncological references in Brazil (Clinical Hospital of the State University of Campinas - UNICAMP, A.C. Camargo Cancer Center - SP and Santa Casa de Sao Paulo). All surgeries were performed by urology residents under staff supervision. This research was approved by the Ethics Committee of UNICAMP Medical School.

Pre-operative evaluation included physical examination, laboratorial evaluation of hemoglobin/hematocrit, platelet count, renal and coagulation functions and cardiac/respiratory assessments. Regarding imaging evaluations, most patients were diagnosed by abdominal/pelvic ultrasonography or computed tomography. However, in some cases in which the previous images were inconclusive, diagnosis of the $\mathrm{BCa}$ was made by standard white-light cystoscopy (WLC).

The majority of the procedures were performed under epidural anesthesia but a few, due to anesthetics contraindication to regional, occurred under general anesthesia; all in lithotomy position. The surgical equipment was a $26 \mathrm{Fr}$ monopolar resector and the liquid used for bladder irrigation was Glycine or sorbitolmannitol solution. All TURBT were in primary bladder tumors and were considered complete (no macroscopic tumor left). They were performed in two steps: first, the exophytic part of the NMIBC was removed and its fragments evacuated; next, in a similar manner, the deeper layers were resected, including underlying bladder wall with the detrusor muscle and the 
edges of the resection area. The fragments were referred to the pathologist in separate containers.

At the re-TURBT, our data regards the oncological positivity exclusively of the cicatricial area and the specimen was dispatched in only one container. During the cystoscopy all bladder was evaluated looking for others tumors or suspected lesions. Bladder random biopsies weren't performed in any of the cases.

At the end of both procedures TURBT and reTURBT, patients were left with a 3-way Foley catheter with continuous saline bladder irrigation.

Each center had a different genitourinary specialist pathologist performing the pathological analysis of the tissues collected separately in each institution.

All data were collected from patients' records of those three institutes and included: demographic characteristics such as age, gender, the dates of the TURBT and re-TURBT and the histopathological samples results of both surgeries.

Overall, the inclusion criteria were: complete macroscopical initial TURBT and presence of MP in the pathological material of the first surgery; the indication of reTURBT based precisely at the histological EAU guidelines, such as the detection of a high-grade NMIBC and/or a $\mathrm{T} 1$ at initial resection. Because of the large number of patients waiting for this procedure, in these three major centers, we allowed the period between the first and the second surgeries to be 12 weeks, longer than the 6 weeks advocated by most guidelines.

The following exclusion criteria were used: patients with preoperative radiographic images suggesting muscle-invasive disease, known muscle-invasive tumor at first TURBT, those for whom the surgeon documented "incomplete macroscopic BCa resection", whose pathological analysis did not show MP fragments or those that were inconclusive to determine which specific muscle bundle was resected, or patient submitted to the re-TURBT after more than 12 weeks.

\section{Results}

We identified sixty-six patients who met the inclusion criteria, 50 male $(75,8 \%)$ and 16 female $(24,2 \%)$. The median age was 64 years (range 39 to 88). The median age of the patients whose re-TURBT identified residual tumor was
65 years (range to 42 to 88 ), while in the negative group, the median age was 63 years (range 39 to 88). Women had residual cancer in 3 cases $(18.7 \%)$ and men in $11(22 \%)$. (Table 1$)$

Table1. Histopathological positivity of the reTURBT according to age and gender

\begin{tabular}{|c|c|c|c|}
\hline & $\begin{array}{l}\text { Re- } \\
\text { TURBT } \\
\text { Negative }\end{array}$ & $\begin{array}{l}\text { Re- } \\
\text { TURBT } \\
\text { Positive }\end{array}$ \\
\hline \multicolumn{2}{|c|}{ Patients (\%) } & $52(78.8 \%)$ & $14(21.2 \%)$ \\
\hline \multicolumn{2}{|c|}{ Age (median/range) } & $63(39-88)$ & $65(42-88)$ \\
\hline \multirow[t]{2}{*}{ Gender } & Female(\%) & $39(78 \%)$ & $11(22 \%)$ \\
\hline & Male (\%) & $13(81.3 \%)$ & $3(18.7 \%)$ \\
\hline
\end{tabular}

Oncological free-status was histologically confirmed in $52(78.8 \%)$ at the reTURBT, whereas residual tumor was detected in 14 $(21.2 \%)$ of the 66 patients at the second surgery.

After complete resection in the first TURBT, all residual disease in the re-TURBT was detected in the cicatricial area of initial tumor. No tumor or suspicious lesion was observed in other areas of the bladder.

Regarding the 14 patients who were diagnosed with residual tumors in the re-TURBT, 12 had a T1 high grade on the histological analysis of the first resection, and 2 had a T1 low grade. No patients with a pTa high grade at the initial TURBT presented cancer at pathological analysis of the second resection. (Table 2) .

Table2. Histopathological positivity of the reTURBT according to the histopathological analysis of the initial TURBT

\begin{tabular}{|l|l|l|l|}
\hline & $\begin{array}{c}\text { Re- } \\
\text { TURBT } \\
\text { Negative }\end{array}$ & $\begin{array}{c}\text { Re-TURBT } \\
\text { Positive } \\
\text { (Same stage) }\end{array}$ & $\begin{array}{c}\text { Re-TURB T } \\
\text { Positive } \\
\text { (Upstaged - } \\
\text { pT2) }\end{array}$ \\
\hline $\begin{array}{l}\text { Initial TURBT } \\
\text { pTa high grade }\end{array}$ & $5(100 \%)$ & 0 & 0 \\
\hline $\begin{array}{l}\text { Initial TURBT } \\
\text { pT1 low grade }\end{array}$ & $7(77.8 \%)$ & $2(22.2 \%)$ & 0 \\
\hline $\begin{array}{l}\text { Initial TURBT } \\
\text { pT1 high grade }\end{array}$ & $40(77 \%)$ & $11(21.1 \%)$ & $1(1.9 \%)$ \\
\hline
\end{tabular}

One patient of the 14 who had residual tumor $(1.5 \%)$ also had his disease upstaged (T1 high grade to T2) and so this pathological finding altered the proposed therapeutic regimen to an unequivocal indication of cystectomy in a 59 year-old patient. Therefore, the restaging resection resulted in an indication of a new approach in one case. All of the remaining 13 patients had the same disease stage in the reTURBT as the first resection's stage. 


\section{DISCUSSION}

In the ICUD-EAU International Consultation on Bladder Cancer - 2012, the authors reviewed data on re-TURBT for all T1 tumors and concluded that the chance of finding residual cancer on re-resection within 4 weeks of the initial TURBT for a pT1 is $30 \%$, but it does not specify the grade of the $\mathrm{T} 1$ or if they only consider "residual" tumors at the primary site $[2,5]$. Nevertheless, at the conclusion of a prospective randomized clinical trial at European Urology, authors affirm that several studies published before included in the analysis of re-TURBT patients that even had macroscopic residual tumors. Moreover, Divrik et al considered there was microscopic oncological positivity in $33.8 \%$ of the cases submitted to a second TURBT. However, out of the 27 patients in this group, only 12 had cancer detected at the primary site, whereas 11 had elsewhere; and 4 had remaining tumor at the site of the first resection and in a different site. Also, it makes another important conclusion that the risk of having a residual NMIBC directly correlates with the progressive grade of the initial cancer: $\mathrm{G} 1, \mathrm{G} 2$ and $\mathrm{G} 3$ (p=0.009) [11]. In our study, only 2 patients with residual tumor had a previous pT1 low grade cancer initially resected, but without a significant difference from the pT1 high grade, respectively $22.2 \%$ and $23 \%$.

Furthermore, we consider essential to discuss the quality of the initial surgery. Herr and Donat reiterated that this might be measured by determining completeness of resection, ability to obtain MP for analysis and recurrence at the resection site [12]. Moreover, Soloway et al [13], in a retrospective study, observed that a substantial number of "early recurrences" are due to $\mathrm{BCa}$ not being resected rather than true recurrence of NMIBC. They also make some recommendations to improve the TURBT, such as: use of a $70^{\circ}$ lens, careful review of the entire surface of the bladder, cystoscopy survey with the bladder almost full and with approximately $25 \%$ capacity and documentation of all abnormalities before resection to plan a detailed resection.

The depth of the resection and to be sure of the complete resection are the most important steps to be achieved in the initial TURBT. These could have impacted in the results, once our revision showed $21.2 \%$ of positivity and had only one case of upstage in the re-TURBT pathological findings, a little lower rate than usually stated in the literature. We believe that many studies report a higher percentage of oncological positivity on re-TURBT, because they consider incomplete resection as a "residual cancer". We also agree with Kolozsy when he affirms that the main area of residual tumor is at the base of the crater remaining at the tumor site [14], once all patients with residual disease in this study had detection in the cicatricial area of initial tumor.

Yet about surgical technique, in a study published in 2010, an Uro-Oncology group from the United Kingdom observed the presence or absence of MP in the initial resection, and concluded that the lack of this layer at the pathological evaluation is an independent predictor to residual disease at early re-TURBT. It also compared $\mathrm{BCa}$ resections carried out by juniors and seniors trainees and it observed that the surgery performed by the less experienced surgeons independently predicted a higher recurrence rate at first follow-up cystoscopy [15]. We did not separate our procedures in different groups according to which resident performed the surgery.

Several technological innovations have appeared to improve urologist's ability to perform a more complete TURBT, including photodynamic diagnosis (PDD) cystoscopy and narrow-band imaging (NBI). PDD, also known as fluorescence cystoscopy, facilitates visualization of bladder tumors compared with standard WLC, detection rates of 73-96\% with WLC alone versus 90-96\% with PDD, according to some studies. This new technique can be especially helpful in the detection of CIS - 23-68\% with WLC alone compared with 91$97 \%$ with PDD. It is also associated with reduced rates of residual $\mathrm{BCa}$ at first check cystoscopy and, in some series, decreased recurrence rates $[3,5]$. PDD and NBI may be essential tools in the future to help us minimize the need of a second intervention, if we can also prove that they leave fewer tumors behind after the first resection, not only at different locations in the bladder, but also residual cancer at the original resection site.

Herr advocates about the importance of the reTURBT in achieving two goals: distinguishing understaged muscle-invasive bladder cancer from real pT1 tumors and early indicating the need for a cystectomy. Our study had one case of a T2 tumor that had a histological stage of T1 
high-grade in the first resection and, therefore, the re-TURBT indicated the need for an immediate cystectomy [16].

Moreover, Herr states that patients who have minimally invasive tumors on the re-TURBT often have more advanced disease at cystectomy. In a study of 57 patients with T1 disease who underwent radical cystectomy, Stenberg et al. found $25 \%$ of muscle invasive (T2) tumors, whereas Ark et al. had $45 \%$ of muscle invasive tumors diagnosed in immediate cystectomy of patients who had $\mathrm{T} 1$ histological tumors on re-TURBT. Interestingly, Herr also defends that that the upstaged disease often found in the immediate cystectomy of patients who had pT1 tumors at re-TURBT justify the non-conservative approach for these patients after re-TURBT, against the intravesical BCG therapy [17-19] had we followed these recommendations 13 other patients would have undergone cystectomy, resulting in a major increase in the number of cystectomies after reTURBT.

On the other hand, Villavicencio et al in 2012 [20] affirm that a re-TURBT could not be necessary in all the high-grade NMIBC. They defend that the restaging surgery is only obligatory if there is absence of MP in the TURBT's fragments. If the initial resection was complete, these authors performed the reresection on particular characteristics of the pTa or pT1, such as size, multiplicity and localization. The results of their paper in these cases were very similar to ours considering the analysis of residual tumor at the re-TURBT: $17.1 \%$, compared to $21.2 \%$ in this study. Interestingly, they also discussed that the incidence of complications in the initial TURBT varies from 5.7 to $9.9 \%$, while in the second surgery increases to $12.6 \%$, probably due to a thinner vesical wall secondary to the first resection. They included urinary tract infection, urethral stenosis, continuous bleeding or bladder perforation and some imperative of re-operation. Moreover, the indication of a re-TURBT adds considerable economic costs for the health system to treat this frequent disease.

In our understanding, this is the most important casuistic published from re-TURBT in Brazil and comparable to many other studies in literature [21-23].

\section{Conclusions}

We observed that the positivity of oncological findings was not so high like in previous studies on the re-TURBT, was detected only at the primary tumor site and did not change dramatically the therapeutic strategy, in spite of the retrospective design and the small number of patients reviewed in this study.

In the future, complete resection of the tumor at the first resection, better choice of these patients to re-TURBT and the use of new technologies can diminish the indications of the restaging resection. These improvements could decreasing the costs and risks inherent to this surgery. Further studies are necessary to corroborate our statements.

\section{REFERENCES}

[1] Kamat AM, Hegarty PK, Gee JR, Clark PE, Svatek RS, Hegarty N et al (2013) ICUD-EAU International Consultation on Bladder Cancer 2012: Screening, diagnosis, and molecular markers. Eur Urol 63(1):4-15.

[2] Soloway MS (2013) ICUD-EAU International Consultation on Bladder Cancer 2012: Recommendations on bladder cancer-progress in a cancer that lacks the limelight. Eur Urol 63(1):1-3.

[3] Tilki D, Burger M, Dalbagni G, Grossman HB, Hakenberg OW, Palou J, et al (2011) Urine markers for detection and surveillance of nonmuscle-invasive bladder cancer. Eur Urol 60(3):484-92.

[4] Pompeo ACL, Wroclawski ER, Sadi MV (2007) Carcinoma urotelial da bexiga. In:Pompeo ACL, Wroclawski ER, Sadi MV (eds) Algoritmos em Uro-Oncologia. Rio de Janeiro, Brasil. pp 92-116.

[5] Burger M, Oosterlinck W, Konety B, Chang S, Gudjonsson S, Pruthi R, et al (2013) ICUDEAU International Consultation on Bladder Cancer 2012: Non-muscleinvasive urothelial carcinoma of the bladder. Eur Urol 63(1):3644.

[6] Amin MB, Smith SC, Reuter VE, Epstein JI, Grignon DJ, Hansel DE, et al (2015) Update for the practicing pathologist: The International Consultation On Urologic Disease-European association of urology consultation on bladder cancer. Mod Pathol 28(5):612-30.

[7] Soloway MS (2007) It is time to abandon the "superficial" in bladder cancer. Eur Urol 52(6): 1564-5.

[8] Amin MB, McKenney JK, Paner GP, Hansel DE, Grignon DJ, Montironi R, et al (2013) ICUD-EAU International Consultation on 
Bladder Cancer 2012: Pathology. Eur Urol 63(1):16-35.

[9] Dalbagni G, Herr HW, Reuter VE (2002) Impact of a second transurethral resection on the staging of $\mathrm{T} 1$ bladder cancer. Urology 60(5):822-4; discussion 824-5.

[10] Maruniak NA, Takezawa K, Murphy WM (2002) Accurate pathological staging of urothelial neoplasms requires better cystoscopic sampling. J Urol 167(6):2404-7.

[11] Divrik T, Yildirim U, Eroğlu AS, Zorlu F, Ozen H (2006) Is a second transurethral resection necessary for newly diagnosed pT1 bladder cancer? J Urol 175(4):1258-61.

[12] Herr HW, Donat SM (2008) Quality control in transurethral resection of bladder tumours. BJU Int 102(9 Pt B):1242-6.

[13] Adiyat KT, Katkoori D, Soloway CT, De los Santos R, Manoharan M, Soloway MS (2010) "Complete transurethral resection of bladder tumor": are the guidelines being followed? Urology 75(2):365-7.

[14] Kolozsy Z (1991) Histopathological "self control" in transurethral resection of bladder tumours. Br J Urol 67(2):162-4.

[15] Mariappan P, Zachou A, Grigor KM (2010) Detrusor muscle in the first, apparently complete transurethral resection of bladder tumour specimen is a surrogate marker of resection quality, predicts risk of early recurrence, and is dependent on operator experience. Eur Urol 57(5):843-9.

[16] Herr HW (2015) Role of repeat resection in non-muscle-invasive bladder cancer. J Natl Compr Canc Netw 13:1041-6.
[17] Herr, HW (2016) Counterpoint: Is Immediate Radical Cystectomy Justified When NonMuscle-Invasive Bladder Cancer First Presents as High-Grade T1 Urothelial Carcinoma on ReResection? Oncology 30(6): 543-4.

[18] Sternberg IA, Keren Paz GE, Chen LY, et al (2013) Role of immediate radical cystectomy in the treatment of patients with residual $\mathrm{T} 1$ bladder cancer on restaging transurethral resection. BJU Int 112:54-9.

[19] Ark JT, Keegan KA, Barocas DA, et al (2014) Incidence and predictors of understaging in patients with clinical $\mathrm{T} 1$ urothelial carcinoma undergoing radical cystectomy. BJU Int 113:894-9.

[20] Gaya JM, Palou J, Cosentino M, Patiño D, Rodríguez-Faba O, Villavicencio H (2012) La re-resección transuretral puede no ser necesaria en todos los tumores vesicales no músculoinvasivos de alto grado. Actas Urol Esp 36(9):539-544.

[21] Gendy R, Delprado W, Brenner P, Brooks A, Coombes G, Cozzi P et al (2016) Repeat transurethral resection for non-muscle-invasive bladder cancer: a contemporary series. BJU Int117(4):54-9.

[22] Hashine K, Ide T, Nakashima T, Hosokawa T, Ninomiya I, Teramoto N (2016) Results of second transurethral resection for high-grade T1 bladder cancer. Urol Ann8(1):10-5.

[23] Shim JS, Choi H, Noh TI, Tae JH, Yoon SG, Kang SH et al (2015) The clinical significance of a second transurethral resection for T1 highgrade bladder cancer: Results of a prospective study. Korean J Urol56(6):429-34.

Citation: Ubirajara Ferreira, Wagner E. Matheus, Wilmar Azal Neto, Marcelo A. Souza, Roni C. Fernandes, Bruno N. Calado et al. Multicenter Analysis of the Oncological Positivity on Restaging Resection (Re-Turbt) of Urothelial Non-Muscle-Invasive Bladder Cancer. ARC Journal of Urology.2017; 2(2):25-30 doi: dx.doi.org/10.20431/2456-060X.0202004

Copyright: (C) 2017 Authors. This is an open-access article distributed under the terms of the Creative Commons Attribution License, which permits unrestricted use, distribution, and reproduction in any medium, provided the original author and source are credited. 